THE JOURNAL OF

THORACIG

GARDIOVASCULAR

SUREERY

Vol. 136, No. 3, September 2008

\title{
Seeking a Higher Standard for Degenerative Mitral Valve Repair: Begin with Etiology
}

David H. Adams, MD, and Ani C. Anyanwu, MD

From the Department of Cardiothoracic Surgery, Mount Sinai Medical Center, New York, NY.

Received for publication Oct 8, 2007; accepted for publication Oct 19, 2007.

Address for reprints: David H. Adams, MD, Professor and Chairman, Department of Cardiothoracic Surgery, Mount Sinai Medical Center, 1190 Fifth Avenue, New York, NY 10029, Telephone 212659 6820, Fax 2126596818 (E-mail: david. adams@mountsinai.org).

J Thorac Cardiovasc Surg 2008;136:551-6 $0022-5223 / \$ 34.00$

Copyright (C) 2008 by The American Association for Thoracic Surgery

doi:10.1016/j.jtcvs.2007.10.060

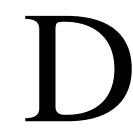
espite the widely held consensus that valve repair is the preferred surgical treatment for patients suffering from degenerative mitral valve disease, valve replacement for this condition remains all too prevalent. In the past few years interest in mitral valve repair has expanded among cardiologists and surgeons, with the recognition that asymptomatic patients with severe mitral regurgitation may be candidates for surgery provided they are likely to undergo valve repair and obtain a durable result. We address both issues in the context of a recent article published in the Journal, which explored the results of mitral valve repair in degenerative disease according to etiologic classification - Barlow's disease or fibroelastic deficiency. ${ }^{1}$ Most reports in the mitral valve repair literature define patient subsets on the basis of leaflet dysfunction (posterior, anterior or bileaflet prolapse) and repair techniques (chordal shortening or artificial chordoplasty; annuloplasty ring or no annuloplasty ring, etc.), without clarification of the etiology of degenerative disease. Furthermore, these studies traditionally used patient survival and freedom from re-operation as the principal indicators of a durable result. ${ }^{2-4}$ Recently, however, it has been appreciated that a proportion of patients free from reoperation after mitral valve repair have significant recurrent mitral regurgitation ${ }^{5,6}$ implying that freedom from reoperation is not a robust measure of durability of mitral valve repair.

In their recent article, Flameng and co-workers ${ }^{1}$ introduced a fresh dimension into mitral valve repair outcomes research, by attempting to define the long-term outcome of mitral valve repair, including the freedom from recurrent mitral regurgitation, on the basis of etiology of degenerative mitral valve disease. Their data suggest, perhaps surprisingly, that, provided the surgical techniques were optimal, patients have a similar rate of recurrent regurgitation after mitral valve repair regardless of whether the original disease was Barlow's or fibroelastic deficiency. There are several limitations in their analysis which deserve emphasis and suggest the need for further study, including retrospective classification of etiology and non-standardized and evolving surgical techniques - both of which limit the robustness of outcomes data. ${ }^{7}$ Nonetheless, this emphasis on etiologic classification in outcomes analysis of degenerative mitral valve repair is significant. We believe accurate etiologic classification is crucial to outcomes research, ${ }^{7}$ and indeed to achieving a higher standard of clinical care, ${ }^{8}$ as it does not seem logical that degenerative valves with very diverse characteristics (Figure 1a, 1c) are considered the same.

\section{What are the Differences in Degenerative Etiology?}

We previously highlighted the importance of etiologic distinction in the field of degenerative mitral valve repair. ${ }^{8}$ The surgical classification of degenerative mitral 

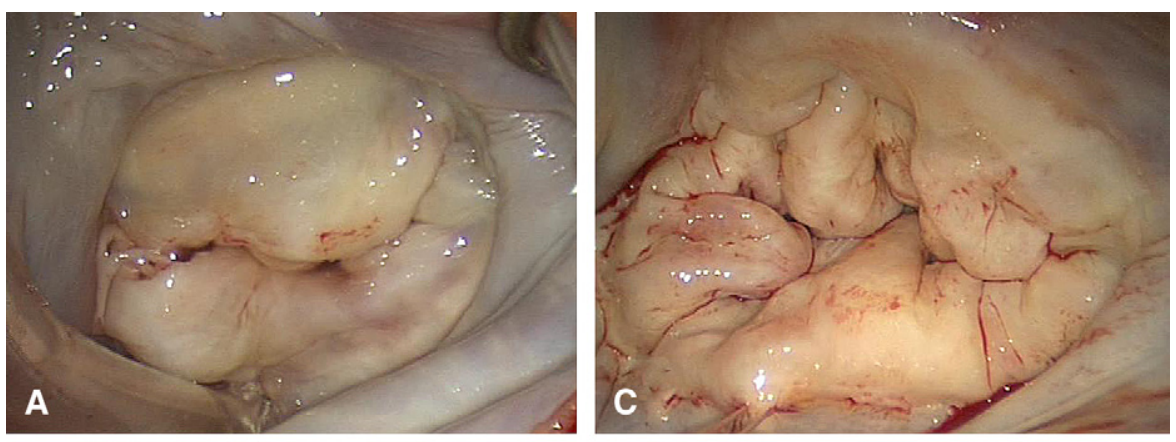

Figure 1. Etiologic comparison of degenerative mitral valve disease: Fibroelastic deficiency resulting in chordal rupture with single segment prolapse [P1] (a). This valve was repaired with a limited triangular resection and ring annuloplasty (b). In contrast, Barlow's disease (c) is characterized by multisegment prolapse and marked excess tissue; repair technique included extensive resection, annular plication, slid-
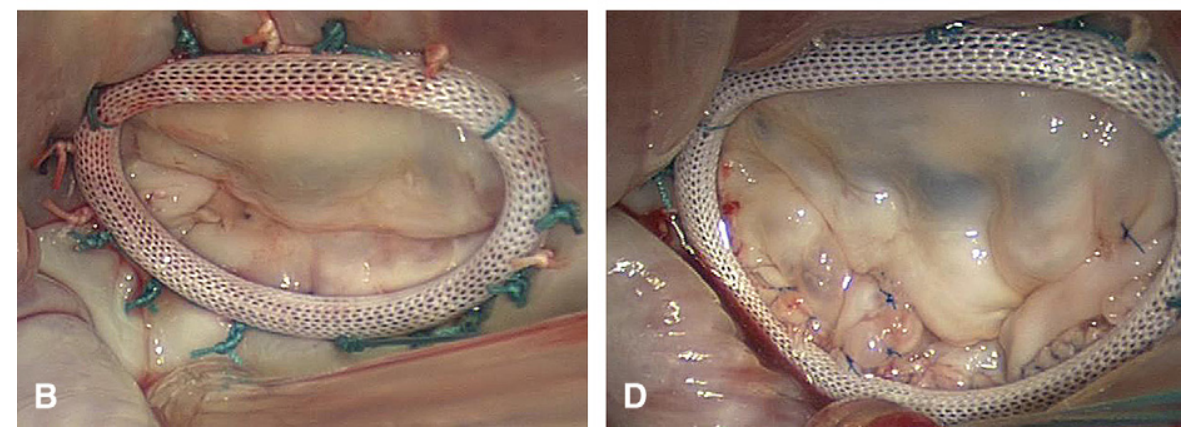
ing plasty, commisuroplasty and large ring annuloplasty (d).

valve disease is based predominantly on the pioneering work of Carpentier and colleagues who defined the key differentiating factors of these conditions in the 1970s. Barlow's disease is often evident by early adulthood, and most patients have been aware of a murmur or valve disease for several years. They usually present with indications for surgery in their fifth or sixth decade of life. Barlow's disease is characterized by myxomatous degeneration affecting the entire valve, resulting in excess leaflet tissue, leaflet billowing and multi-segmental prolapse and/or distension (Figure 1c). Echocardiography usually shows a large valve size with excess multi-segmental tissue and billowing of the body of the leaflet(s). Late systolic prolapse in the setting of chordal elongation may be observed or, conversely, early systolic prolapse in the setting of ruptured chordae. Calcification, leaflet restriction due to chordal fibrosis, and posterior leaflet hinge displacement away from the atrioventricular junction may be evident. In contrast fibroelastic deficiency is a disease typically of older individuals over the age of 60 years with a relatively short history of mitral valve disease. Fibroelastic deficiency is essentially a chordal disease in which presumed connective tissue deficiency predisposes to chordal rupture, typically resulting in single segment prolapse (Figure 1a). In contrast to Barlow's disease, the leaflets are generally thin and without excess tissue except in the prolapsing segment which may be distended with myxomatous changes. ${ }^{9}$ Usually, the diagnosis can be made on pre-operative echocardiography which suggests a normal sized valve with thin leaflets and minimal excess tissue and isolated chordal rupture.

Carpentier's group was the first to note that while these two etiologic differentiations of degenerative disease can be made in a majority of patients, a clear distinction between Barlow's disease and fibroelastic deficiency is not possible in up to $20 \%$ of patients, even when surgical and histological findings are taken into consideration. ${ }^{9}$ Etiologies unclassifiable into either group include systemic connective tissue disorders, formes frustes of Barlow's disease, senile degeneration, dystrophic calcification and idiopathic degeneration. ${ }^{9}$ Indeed Flameng et al noted in a prior article that "a clear distinction remains difficult" between Barlow's disease and fibroelastic deficiency, ${ }^{6}$ so it is somewhat surprising that they were able to classify all cases as Barlow's disease or fibroelastic deficiency in the current series. ${ }^{1} \mathrm{We}$ find patients with an intermediate valve size (32 to 34 $\mathrm{mm}$ ) and a very distended P2 segment notoriously difficult to classify. By forcing all patients into either Barlow's disease or fibroelastic deficiency, Flameng et al $^{1}$ likely misclassified a significant proportion of their patients. In our recent Barlow's series, ${ }^{10}$ we chose to restrict our definition of Barlow's disease to patients receiving a $36 \mathrm{~mm}$ or greater ring, in order to define a pure Barlow's subgroup with minimal potential of contamination bias from misclassified fibroelastic deficiency cases. In the recent report by Flameng and co-authors, ${ }^{1} 40 \%$ of patients classified as having Barlow's disease received a ring size of $32 \mathrm{~mm}$ or less; such 
small valve size is not consistent with true Barlow's disease and it is likely that this group included several patients with fibroelastic deficiency who were wrongly classified as Barlow's disease. This potential misclassification must be taken into context when considering the implications of their study.

\section{What are the Surgical Implications of Degenerative Etiology?}

The etiology of degenerative mitral valve disease has several important surgical implications which deserve emphasis. Degenerative etiology influences the type and complexity of lesions the surgeon will encounter in the operating room, which in turn determines the specific techniques required to achieve a successful repair. Taking this a step further, because different techniques require different levels of surgical expertise and experience, etiology has a direct implication on choice of surgeon to perform the operation in a particular patient. It is therefore imperative that prior to surgical intervention, the cardiologist and cardiac surgeon make an effort to differentiate the etiology of degenerative disease, as well as the burden of specific segmental prolapse and lesions, in order to maximize the likelihood of repair.

\section{Operative Strategy}

The etiology of degenerative mitral valve disease has an impact on the complexity and number of repair techniques required to achieve a successful valve repair, mainly because of the difference in burden of lesions encountered. ${ }^{8}$ In fibroelastic deficiency, there is most commonly a single lesion (chordal rupture) resulting in a single segment prolapse (Figure 1a), usually the P2 segment of the posterior leaflet. Since the non-prolapsing segments are usually void of excess leaflet tissue, a repair can typically be undertaken by simple resection (quadrangular or triangular) of the unsupported leaflet segment (as in Figure 1b). Alternatively, transfer of secondary chordae or application of artificial chords can be used to correct a marginal prolapse without leaflet resection. As all these techniques are relatively straightforward, it is not surprising that current repair rates for simple cases of fibroelastic deficiency approach $100 \%$ in most tertiary mitral surgical centers. Furthermore, with simplified approaches such as limited triangular resections, ${ }^{11}$ most posterior leaflet prolapse due to fibroelastic deficiency should be repairable by experienced general cardiac surgeons.

In contradistinction, Barlow's disease often presents several lesions coexisting in multiple segments of the same valve, which may include chordal elongation, chordal rupture, chordal fusion and fibrosis, excess leaflet tissue, posteror leaflet displacement, annular or papillary muscle calcification, and annular dilatation. The surgical repair approach should address all lesions present whenever possible. This often requires advanced repair techniques such as exten- sive leaflet resection, leaflet detachment from the annulus, sliding-plasty to lower the height of remaining posterior leaflet segments, multiple chordal transfers or multiple artificial chordae, and large annuloplasty rings (Figure 1d). Particular attention must be paid to the amount of residual leaflet tissue and the annular dimensions after annuloplasty to avoid the occurrence of systolic anterior motion. A repair of this nature (as in Figure 1d) predictably requires more skill and expertise than a repair for simple chordal rupture in fibroelastic deficiency (as in Figure 1b). Although we have found it possible to also achieve repair rate approaching $100 \%$ for Barlow's valve disease,${ }^{10}$ most series suggest a lower repair rate for Barlow's compared to fibroelastic disease. This is evident even in expert series, such as in Flameng et al's recent series, where their contemporary repair rate for a Barlow valve was $85 \%$ compared to $100 \%$ for fibroelastic deficiency. ${ }^{1}$

\section{Who Should Perform the Surgery?}

There is a general consensus in guidelines that mitral valve repair is the preferred treatment of degenerative valve disease, and that in comparison to mitral valve replacement procedures, it is associated with improved survival in most patient cohorts. ${ }^{12}$ Although mitral valve repair rates have been gradually rising over the past decade, uptake in some centers remains surprisingly low, especially considering that the majority of techniques used in valve reconstruction have been well established since the 1980 's. ${ }^{13}$ Clearly the issues behind the underutilization of mitral valve repair are multi-factorial and the path to making it a routine procedure that is widely available has proven elusive. Specifically, issues including volume and experience as well as referral patterns deserve close scrutiny. Compared to coronary artery bypass operations and valve replacement procedures, most cardiac centers do relatively few mitral valve repair procedures for degenerative disease; low volume centers have lower repair rates. ${ }^{14}$ In a recent examination of the mitral valve sub-group in the Euro Heart Survey on Valvular Heart Disease, ${ }^{15}$ it was noted that a lack of available expertise (and not the complexity of the lesions) was given as the reason for over a third of valve replacement procedures. In an attempt to address this issue, a multi-disciplinary team in the United Kingdom suggested one way of increasing mitral valve repair rates was to establish "best practice standards" that would result in directed referral to increase volume and experience, and require mitral valve repair surgeons to perform a minimum of 25 repairs a year. ${ }^{16}$ Furthermore, it was proposed that mitral surgeons undergo regular audit, and make not only their repair rates, but also their incidence of residual and recurrent mitral regurgitation publicly available. The authors went on to suggest that patients with bileaflet prolapse or Barlow's disease should be referred to "super sub-specialists" although criteria for such were not defined. ${ }^{16}$ The logic 
of the rationale for such an approach can be appreciated in the recent article by Flameng et al. ${ }^{1}$ Although there was an increase in the overall repair rate with accumulating experience over time, their rate of repair for Barlow's disease was always lower than that for fibroelastic deficiency, reinforcing the complexity of repair in this subgroup.

We believe the next logical step is to recommend interhospital or inter-surgeon comparison of the process and outcomes of degenerative mitral valve repair including repair rate, residual or recurrent mitral regurgitation, and reoperation for failed valve repair in clearly defined etiologic sub-groups. Taking into consideration that the patient with Barlow's disease is generally younger, he or she has the most to benefit from valve repair, as opposed to replacement, so given the anticipated complexity of repair in such patients, a documented repair rate for this condition should be made available to these patients as part of the informed consent. Many experienced cardiac surgeons will have a success rate in excess of $90 \%$ for simple $\mathrm{P} 2$ prolapse in the setting of fibroelastic deficiency, but have a low repair rate for Barlow's valves. Requiring surgeons undertaking mitral repair surgery to perform at least 25 repairs a year, ${ }^{16}$ does not go far enough, as etiology and lesion complexity, rather than volume alone, is critical to understanding the true repair rates a specific patient can expect from a given surgeon. For example, a surgeon who does 30 mitral valve procedures a year with an $83 \%$ repair rate, could have repaired 10 of 10 ischemic mitral valves cases (100\%), 14 of 15 cases (93\%) of fibroelastic deficiency with posterior leaflet prolapse, but only 1 of 5 cases (20\%) with Barlow's disease, so quoting the $83 \%$ repair rate to a patient with Barlow's would be misleading. Ideally therefore, differentiation between Barlow's and fibroelastic deficiency, as well as simple vs. complex lesions, should take place pre-operatively as a surgical team should only undertake an operation on a patient who requires a complex repair if it is well versed in the advanced techniques needed to achieve a complex valve reconstruction. The referring cardiologist should make a specific attempt to define the likelihood of repairability of the valve based on the etiology and complexity of lesions seen on echocardiography and the surgical experience in their own center. ${ }^{17} \mathrm{~Pa}-$ tients with a projected low likelihood of repair in their local center, especially the young Barlow patient, should be referred to a regional reference center with an more established track record in complex repair. Likewise, the surgeon should give an honest assessment of the likelihood of a achieving a successful repair based on their own experience with the etiologic group and specific lesions in the patient being considered for surgery. If the honest expectation, based on volume and expertise, is that there is a good chance a valve replacement will be required for the degenerative disease encountered, the surgeon should be open about this with the patient, and consider offering a second opinion with a more experienced valve repair surgeon.

\section{Implications for Future Outcome Studies}

Data from Flameng and associates ${ }^{1}$ suggest that when adjustment has been made for 'surgical risk factors', patients have a similar linearized rate of recurrent regurgitation after mitral valve repair regardless of whether the original disease was Barlow's (2.9\% per year) or fibroelastic deficiency (2.2\% per year). Because of aforementioned limitations, especially with the classification approach and surgical methods employed, we cannot be certain that this observation is valid. The long term freedom from moderate or severe regurgitation was certainly disappointing in Flameng's series (35\% having moderate or severe regurgitation at 10 years $^{1}$ ) and is worse than reported in a contemporary series from David's group (19\% at 10 years ${ }^{18}$ ). Use of diverse surgical techniques and an inevitable learning curve in the early part of any series (evident in Flameng et al's study by an initial low repair rate for Barlow's disease which increased over the study period ${ }^{1}$ ), could contribute to a high rate of recurrent regurgitation as patients operated in the early part of a series are those that have the long-term follow-up. ${ }^{7}$ Inferior surgical techniques may contribute to inferior long-term outcome. For example, chordal shortening, employed in $15 \%$ and the non-use of an annuloplasty ring in 5\% of Barlow repairs in Flameng et al's series, ${ }^{1}$ are predictors of recurrent mitral regurgitation. ${ }^{1,6}$ In reality, we still do not know if the longevity of a Barlow repair differs from that of repair for fibroelastic deficiency. There are data suggesting that bileaflet and anterior leaflet repairs are less durable than posterior leaflet repairs ${ }^{2,5}$ - as bileaflet prolapse is more commonly associated with Barlow's, one would, therefore, expect that more Barlow repairs would fail in the long term than repairs for fibroelastic deficiency. However, we do not know whether there is a difference in durability of repair between similar dysfunctions of different etiology (e.g. posterior leaflet prolapse in Barlow's versus posterior leaflet prolapse in fibroelastic deficiency). We suspect that the two diseases are indeed different: even after successful reconstruction, there remain differences in the reconstructed valve morphology, and in techniques utilized to achieve the repair, (see Figure 1b and 1d) such that the potential for recurrent mitral regurgitation due to technical failure or disease progression would likely differ (with greater likelihood of recurrence in Barlow's disease). Future studies will test this hypothesis. Similarly, when assessing the results of a particular technique, it is important to know what disease the technique has been applied for: the long term results of a single artificial chord to repair P2 prolapse in the setting of fibroelastic deficiency may be different from that of multiple artificial chords used to support extensive posterior leaflet prolapse in Barlow's disease. These questions can only be answered by accurate surgical differentiation of valve lesions and etiology at the time of surgery, near-complete long term follow-up and appropriate 
methods of analysis. ${ }^{7}$ The differentiation of outcome data by etiology will bear increasing relevance in coming years because of the increasing number of patients undergoing surgery for asymptomatic disease, the majority of which will likely have Barlow's disease. In order to counsel patients, we should be aware of the likelihood of long-term recurrence based on the etiology and lesions, and also the techniques employed in the reconstructive procedure.

\section{Learning from Unplanned Mitral Valve Replacement}

Prospective documentation of etiology and lesions is also important for those valves which are replaced for degenerative disease. For example, although the replacement rate for degenerative disease in Flameng et al's recent series was $23 \%,{ }^{1}$ the authors did not provide any information on the replacement group, preventing the reader from learning from the group that had failed or un-attempted repairs. We would recommend that in future studies the specific reasons for valve replacement for degenerative disease should be scrutinized. Additionally, we suggest that surgeons record in the operative report for all mitral valve replacements, what specific lesion(s) made the valve "irrepairable". This may one day help establish a "repairabilty index or score" which may help in preoperative stratification. Requiring surgeons to document precisely why they replace a valve may also increase repair rates as it would force the surgeon to adopt a lesion and etiology based approach, and additionally provide an important benchmark of quality assessment (by enabling monitoring of the appropriateness of valve replacement). This is particularly relevant in the asymptomatic patient with severe mitral regurgitation with degenerative valve disease, where guidelines suggest at least a $90 \%$ possibility of repair should exist before subjecting an asymptomatic patient to surgery. ${ }^{12}$

\section{Where to go Next?}

We see systematic application of etiologic differentiation as the next chapter in outcomes research in degenerative mitral valve repair. It should no longer be sufficient to describe the outcomes of mitral valve repair in terms of patient survival or freedom from reoperation for a mixed cohort of patients with "degenerative disease", or "mitral valve prolapse", or "floppy valves" as we have done in the past. In this era, we should make an attempt to separate Barlow patients from those with fibroelastic deficiency (recognizing some patients will not be classifiable) and provide readers with clear echocardiographic and surgically derived data on the lesions present and techniques employed to treat them. At some point it will be necessary for our Societies to convene an expert panel to agree on a uniform classification for mitral valve lesions and etiology. With this we can ultimately move to the next level of differentiating degenerative mitral valve disease: offering our patients coun- seling, therapy and informed data on outcomes of surgical intervention based on the specific etiology, lesions and dysfunctions a patient has, rather than solely on leaflet involvement or mixed etiologic experiences. Without question, adoption of etiologic differentiation of degenerative disease by cardiologists and cardiac surgeons will lead to more valve repairs, better quality repairs, and better quality valve outcomes research. We strongly encourage the increased use of etiologic and lesion based differentiation in surgical practice and reporting, as we believe this will herald a new and exciting era of higher quality mitral valve repair and outcomes analysis.

\section{References}

1. Flameng W, Meuris B, Herijgers P, Herregods MC. Durability of mitral valve repair in Barlow disease versus fibroelastic deficiency. $J$ Thorac Cardiovasc Surg. 2008;135:274-82.

2. Braunberger E, Deloche A, Berrebi A, Abdallah F, Celestin JA, Meimoun P, et al. Very long-term results (more than 20 years) of valve repair with carpentier's techniques in nonrheumatic mitral valve insufficiency. Circulation. 2001;104:I8-11.

3. Gillinov AM, Cosgrove DM, Blackstone EH, Diaz R, Arnold JH, Lytle BW, et al. Durability of mitral valve repair for degenerative disease. J Thorac Cardiovasc Surg. 1998;116:734-43.

4. Mohty D, Orszulak TA, Schaff HV, Avierinos JF, Tajik JA, Enriquez-Sarano M. Very long-term survival and durability of mitral valve repair for mitral valve prolapse. Circulation. 2001; 104:I1-7.

5. David TE, Ivanov J, Armstrong S, Christie D, Rakowski H. A comparison of outcomes of mitral valve repair for degenerative disease with posterior, anterior, and bileaflet prolapse. J Thorac Cardiovasc Surg. 2005;130:1242-9.

6. Flameng W, Herijgers P, Bogaerts K. Recurrence of mitral valve regurgitation after mitral valve repair in degenerative valve disease. Circulation. 2003;107:1609-13.

7. Adams DH, Anyanwu A. Pitfalls and limitations in measuring and interpreting the outcomes of mitral valve repair. J Thorac Cardiovasc Surg. 2006;131:523-9.

8. Anyanwu AC, Adams DH. Etiologic Classification of Degenerative Mitral Valve Disease: Barlow's Disease and Fibroelastic Deficiency. Semin Thorac Cardiovasc Surg. 2007;19:90-6.

9. Fornes P, Heudes D, Fuzellier JF, Tixier D, Bruneval P, Carpentier A. Correlation between clinical and histologic patterns of degenerative mitral valve insufficiency: a histomorphometric study of 130 excised segments. Cardiovasc Pathol. 1999;8:81-92.

10. Adams DH, Anyanwu AC, Rahmanian PB, Abascal V, Salzberg SP, Filsoufi F. Large annuloplasty rings facilitate mitral valve repair in Barlow's disease. Ann Thorac Surg. 2006;82:2096-100.

11. Gazoni LM, Fedoruk LM, Kern JA, Dent JM, Reece TB, Tribble CG, et al. A simplified approach to degenerative disease: triangular resections of the mitral valve. Ann Thorac Surg. 2007;83: 1658-64.

12. Bonow RO, Carabello BA, Kanu C, de LA Jr, Faxon DP, Freed MD, et al. ACC/AHA 2006 guidelines for the management of patients with valvular heart disease: a report of the American College of Cardiology/American Heart Association Task Force on Practice Guidelines (writing committee to revise the 1998 Guidelines for the Management of Patients With Valvular Heart Disease): developed in collaboration with the Society of Cardiovascular Anesthesiologists: endorsed by the Society for Cardiovascular Angiography and Interventions and the Society of Thoracic Surgeons. Circulation. 2006; 114.e84-231.

13. Carpentier A. Cardiac valve surgery-the "French correction". J Thorac Cardiovasc Surg. 1983;86:323-37. 
14. Gammie JS, O'Brien SM, Griffith BP, Ferguson TB, Peterson ED. Influence of hospital procedural volume on care process and mortality for patients undergoing elective surgery for mitral regurgitation. Circulation. 2007; 115:881-7.

15. Lung B, Baron G, Butchart EG, Delahaye F, Gohlke-Barwolf C, Levang OW, et al. A prospective survey of patients with valvular heart disease in Europe: The Euro Heart Survey on Valvular Heart Disease. Eur Heart J. 2003;24:1231-43.
16. Bridgewater B, Hooper T, Munsch C, Hunter S, von OU, Livesey S, et al. Mitral repair best practice: proposed standards. Heart. 2006;92: 939-44.

17. Adams DH, Anyanwu AC. The cardiologist's role in increasing the rate of mitral valve repair in degenerative disease. Curr Opin Cardiol. 2008; 23:105-10.

18. David TE. Outcomes of mitral valve repair for mitral regurgitation due to degenerative disease. Semin Thorac Cardiovasc Surg. 2007;19:116-20. 